\title{
Spin-sensitive shape asymmetry of adatoms on noncollinear magnetic substrates
}

\author{
D. Serrate,,${ }^{1,2,{ }^{*}}$ Y. Yoshida,,${ }^{1,3}$ M. Moro-Lagares,${ }^{2}$ A. Kubetzka, ${ }^{1}$ and R. Wiesendanger ${ }^{1}$ \\ ${ }^{1}$ Department of Physics, University of Hamburg, 20355 Hamburg, Germany \\ ${ }^{2}$ INA-LMA and Dpto. Física Materia Condensada, University of Zaraqoza, 50018 Zaragoza, Spain \\ ${ }^{3}$ Institute for Solid State Physics, University of Tokyo, Kashiwa 277-8581, Japan
}

(Received 30 October 2015; published 18 March 2016)

\begin{abstract}
The spin-resolved density of states of Co atoms on a noncollinear magnetic support displays a distinct shape contrast, which is superimposed on the regular height contrast in spin-polarized scanning tunneling microscopy. The apparent atom height follows the well-known cosine dependence on the angle formed by the tip and adatom local magnetization directions, whereas the shape contrast exhibits a sine dependence. We explain this effect in terms of a noncollinear spin density induced by the substrate, which in our case is the spin spiral of the Mn monolayer on $\mathrm{W}(110)$. The two independent contrast channels, apparent height and shape, are identified with the Co magnetization projections onto two orthogonal axes. As a result, all components of the overall atom magnetic moment vector can be determined with a single spin-sensitive tip in the absence of an external magnetic field. This result should be general for any atom deposited on noncollinear magnetic layers.
\end{abstract}

DOI: 10.1103/PhysRevB.93.125424

The control of the spin degree of freedom down to the single-atom limit is nowadays a central research issue [1-7], fostered by the increasing need for miniaturization and lowering power consumption in communication technologies. The most suitable technique to address the problem is spinpolarized scanning tunneling microscopy (SP-STM)[8], which has proven to be an excellent tool to perform single-atom magnetometry [1,6]. The design of magnetic structures with a given functionality calls for atomic-scale engineering by means of atomic manipulation or self-assembly of lowdimensional structures. Both of them can be combined with SP-STM $[6,7,9,10]$. For that reason, getting insight into the underlying physics of SP-STM and extending its range of applications is of crucial relevance. The SP-STM principle is the magneto-conductance effect in magnetic tunnel junctions [8,11-14], and relies on probing the imbalance between majority and minority spins in the local density of states (LDOS) with respect to a particular magnetic quantization axis. Following the same notation as in Ref. [15], the spinpolarized tunneling current at sample bias $V$ reads

$$
I(\mathbf{r}, V, \theta) \propto \rho_{T}\left[\widetilde{\rho}_{s}(\mathbf{r}, V)+P_{T} \tilde{m}_{s}(\mathbf{r}, V) \cos \theta(\mathbf{r})\right],
$$

where $\rho_{T}$ and $P_{T}$ are the tip's density of states and spin polarization, $\tilde{\rho}_{s}$ and $\tilde{m}_{s}$ the integrated sample total density of states and magnetization at $\mathbf{r}$ (tip position), and $\theta$ the angle formed between the tip and local sample magnetization. As a consequence, a SP-STM tip is only sensitive to the projection onto its magnetization direction, $\tilde{m}_{s} \cos \theta$, and not to all other components of $\tilde{\mathbf{m}}_{\mathbf{s}}$. Here we show that the situation is radically different if the substrate's magnetic ground state is noncollinear within the spatial extent of an atomic wave function. Owing to the breaking of translational symmetry in spin space associated with the noncollinearity, the atoms' apparent shapes become distorted in spin-resolved STM images. We find that the strength of such distortion provides a quantitative measurement of the total spin component orthogonal to the default sensitivity direction of the SP-STM probe.

\footnotetext{
*serrate@unizar.es
}

We choose one Mn atomic layer (AL) on W(110) as the supporting substrate for magnetic Co atoms, which exhibits a distinct noncollinear antiferromagnetic (AFM) spin spiral (SS) [16]. Spin-resolved images are taken with a W tip coated with $\sim 50$ AL Fe. The W(110) surface was cleaned by standard cycles of oxygen annealing and flashes to $2000-2100^{\circ} \mathrm{C}$ in ultrahigh vacuum. Designated arrangements of Co atoms on the surface were constructed by lateral atomic manipulation. The measurement temperature is $9 \mathrm{~K}$ unless stated otherwise and image analysis was performed with the WSxM software package [17].

Figure 1 shows constant-current images of a set of Co adatoms on the $\mathrm{Mn} / \mathrm{W}(110)$ antiferromagnetic SS, taken with an out-of-plane magnetized tip. The SS can be described as a $\mathrm{c}(2 \times 2)$ AFM unit cell relative to the $\mathrm{W}(110)$ surface primitive cell, with a deviation off the collinear configuration driven by the Dzyaloshinskii-Moriya interaction [16]. The deviation consists of a local rotation pitch of the spin direction of each $\mathrm{Mn}$ row of about $173^{\circ}$ per primitive unit cell along the

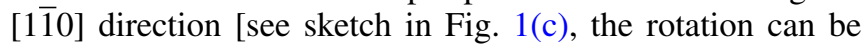
seen as an $\alpha=14^{\circ}$ pitch between equivalent rows of the AFM c(2x2) unit cell]. Co atoms lie on hollow sites and couple ferromagnetically to the Mn row underneath (running parallel to the [001] direction) [9]. Since the SS magnetization samples multiple values of $\theta$, the Co atoms should have different tunneling conductance values in spin-resolved STM images, depending on their position with respect to the SS. This is the origin of the variety of atomic heights in Fig. 1. The change of the Co apparent height in images taken with opposite tip magnetization [cf. Figs. 1(a) and 1(b)] reveals that the height variations are of magnetic origin, reflecting the magnetoconductance of the tip-Co tunnel junction. The height contrast is accompanied by a shape contrast owing to the different orbital characters of the spin-up and spin-down subbands [9], which can be visualized by comparing type-1 and type-2 atoms in Fig. 1(a).

In this experiment, we find three generic types of atoms. For a tip magnetization oriented along the surface normal [Fig. 1(a)], type-1 atoms exhibit a lower apparent height and a more elongated shape than type- 2 atoms, which sit on dark 

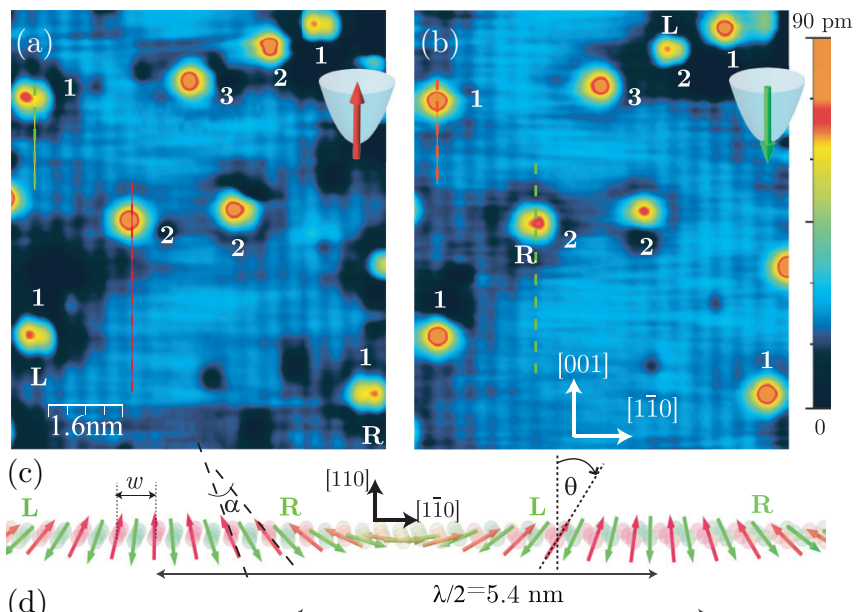

(d)

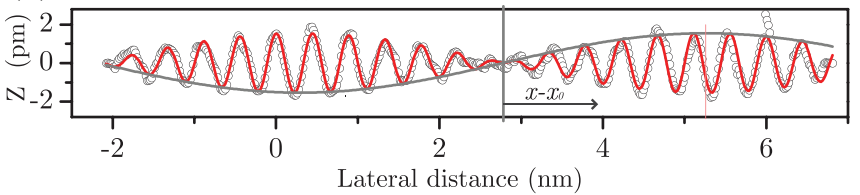

FIG. 1. SP-STM images $(8 \mathrm{mV}, 1 \mathrm{nA})$ of Co atoms on the SS, showing the reversal of the magnetic contrast as the tip magnetization is switched by an external magnetic field of (a) +2 and (b) -2 tesla. Note that the overall surface magnetization is zero, and therefore the external magnetic field does not affect the sample magnetic state, but only controls the tip magnetization, as indicated in the insets. Red (bright Mn row) and green (dark Mn row) dashed lines highlight the half unit cell shift of the magnetic periodicity upon tip magnetization reversal. (c) Sketch of the spin spiral structure, where the red and green color levels of the arrows represent the spin-up and spin-down components, respectively. (d) Experimental spin-resolved profile along the $[1 \overline{10}]$ direction of the spin spiral together with a fit to Eq. (2).

and bright $\mathrm{Mn}$ rows, respectively. If the tip magnetization is oppositely oriented [Fig. 1(b)], type-1 and type- 2 atoms swap their appearance, as the underlying Mn rows do. This indicates that the spin projections of type- 1 and type- 2 atoms $\left[m_{s} \cos \theta\right.$ in Eq. (1)] onto the surface normal have opposite sign. These atoms sit in regions of the SS with large contrast between consecutive Mn rows. According to Eq. (1), such regions correspond to a local nearly AFM arrangement of the $\mathrm{Mn}$ rows with a spin quantization axis mostly out of plane $\left(\theta \simeq 0^{\circ}, 180^{\circ}\right)$, for which $\cos \theta$ takes the maximum difference when $\Delta \theta=180^{\circ}$. There is, however, a third type of atom whose apparent height does not change upon tip magnetization reversal. Type-3 atoms appear in regions with vanishingly small SS AFM contrast. These regions correspond obviously to $\mathrm{Mn}$ rows with a magnetization being nearly in-plane $(\theta \simeq$ $\pm 90^{\circ}$ ), for which $\cos \theta$ is insensitive to $\Delta \theta=180^{\circ}$. Thereby, the spin of type- 3 atoms is mainly in-plane, and has a negligible projection onto the surface normal. It is noteworthy that type-3 atoms do not show any magnetoconductance for a tip probing the out-of-plane spin projection. Conversely, type-1 and -2 atoms would not show magnetoconductance for a tip probing the in-plane projection.

Figures 1(a) and 1(b) contain additional information suggesting that the $\cos \theta$ term in Eq. (1) can be used to probe simultaneously two orthogonal spin directions. The atoms' appearances can be alternatively classified as being higher on the left $(\mathrm{L})$ or on the right $(\mathrm{R})$ side. L- and R-type atoms can be readily identified when located on a dark Mn row, which are represented as green rows in Fig. 1(c), although it is not clear at first sight for those located on bright Mn rows. In the following, we will demonstrate that the shape asymmetry of the spin-resolved Co LDOS provides a quantitative determination of the spin projection orthogonal to the tip magnetization.

In order to establish a relationship between the height/shape of a Co atom and its spin direction, we need a precise and independent measurement of the angle formed between the tip and atom magnetization directions, $\theta_{\mathrm{Co}}$. As Co atoms align their spin parallel to the $\mathrm{Mn}$ row below [9], $\theta_{\mathrm{Co}}$ can be retrieved from the spin-resolved profile of the SS template. Let $w=$ $4.41 \AA$ be the W(110) lattice parameter along [1힐, $\alpha$ the SS rotation per unit cell $\left(\alpha \simeq 14^{\circ}\right)$, and $x_{0}$ the SS position at which $\theta= \pm 90^{\circ}$. As shown in Fig. 1(d), the experimental SP-STM profile of the SS is well described by [16]

$$
z=z_{0}+C \sin \left(2 \pi \frac{x-x_{0}}{w}\right) \sin \left(\frac{\alpha\left(x-x_{0}\right)}{w}\right),
$$

which allows us to extract the angle formed by the magnetization directions of the tip and any Mn row (or adatom on top) at position $x$ as $\theta\left(x_{[1 \overline{1} 0]}\right)-\pi / 2=-\frac{\alpha}{w}\left(x-x_{0}\right)$ with an estimated error of $2.5^{\circ}$.

While the atoms' shape asymmetry was first observed in Ref. [9], Figs. 2(a) and 2(b) unambiguously confirm its systematic variation as a function of $\theta_{\mathrm{Co}}$. This set of atoms was arranged with a spacing of one unit cell (two Mn rows) along [1힝. A fit to Eq. (2) of the SS near the atoms reveals that $\theta_{\mathrm{Co}}$ rotates by $\Delta \theta_{\mathrm{Co}}=-13.5^{\circ}$ between consecutive atoms. Horizontal profiles across the atoms' centers exhibit a gradual increase of shape asymmetry with the trend of enhancing the height on the right side of the atom. This also holds for atoms with dominant spin-up contributions and round shapes $\left(\theta_{\mathrm{Co}}=0^{\circ}\right.$ and $\left.60^{\circ}\right)$, although in this case the asymmetry is less evident and it can only be quantified after a thorough determination of the atom's center position. We derive an upper threshold for the sensitivity to the spin direction of $\sim 14^{\circ}$ just by visual inspection of SP-STM data of the sort of Fig. 2(a). Now, we choose as a measure of the spin shape asymmetry, ShA, the imbalance between the left and right areas encompassed by the constant current profile of the atom, $Z\left(\theta_{\mathrm{Co}}, x_{[1 \overline{1} 0]}\right)$ [Fig. 2(b)].

$$
\begin{gathered}
\operatorname{ShA}\left(\theta_{\mathrm{Co}}, a\right)=\frac{A_{R}-A_{L}}{A_{R}+A_{L}}, \text { where } \\
A_{R[L]}\left(\theta_{\mathrm{Co}}, a\right)=\int_{0[-a]}^{a[0]} Z\left(\theta_{\mathrm{Co}}, x\right) d x .
\end{gathered}
$$

Using several atomic arrangements and four different $\mathrm{Fe}$ coated $\mathrm{W}$ tips, we have characterized the dependence of ShA on $\theta_{\mathrm{Co}}$, displayed in Fig. 3(b). Whereas ShA exhibits a clear $\sin \theta_{\text {Co }}$ behavior, the atom height follows $\cos \theta_{\text {Co }}$ [Fig. 3(a)]. Actually, the height fit is improved if an additional tunneling anisotropic magnetoresistance [18-20] is taken into account with a $\cos ^{2} \theta_{\mathrm{Co}}$ contribution.

Whereas the $\cos \theta_{\text {Co }}$ functional form of SP-STM contrast is well documented in literature $[6,8,12,15]$, the spin-dependent subatomic features are quite surprising. Experimental SP-STM 

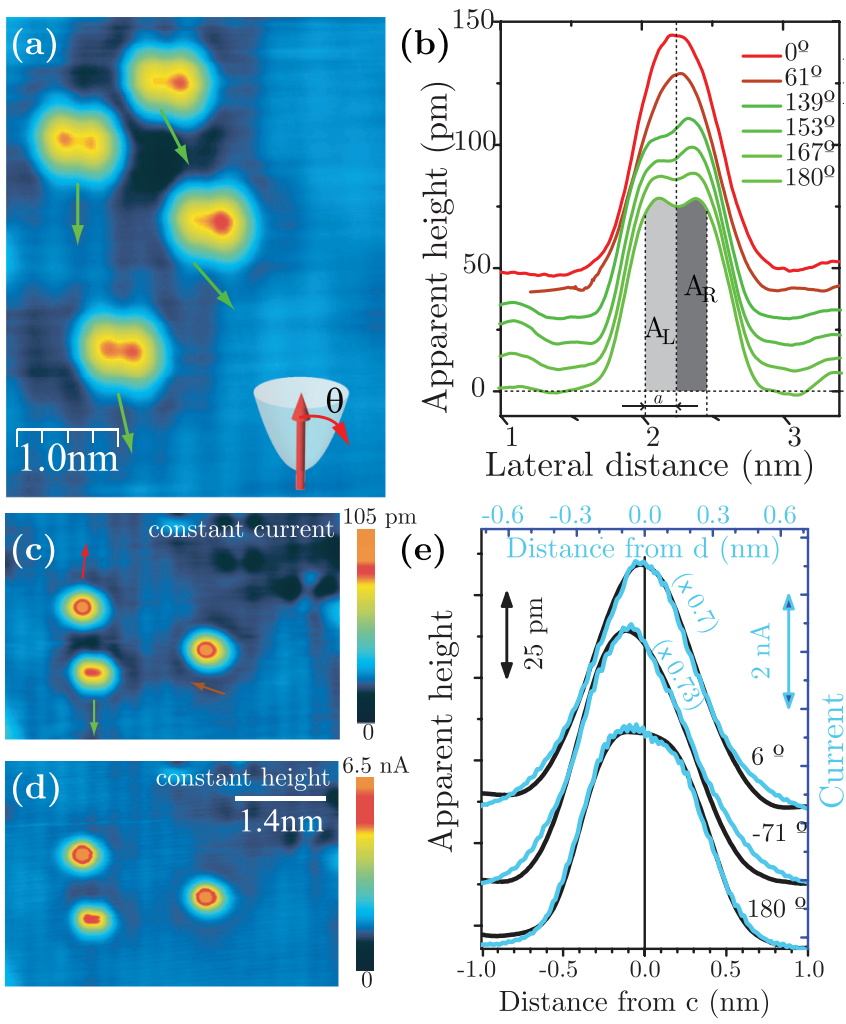

FIG. 2. (a) Set of Co atoms separated by one lattice parameter $(10 \mathrm{mV}, 2 \mathrm{nA}, 2.5 \mathrm{~T})$, showing the gradual increase of the shape asymmetry. This image demonstrates an overall spin direction sensitivity better than $\alpha \simeq 14^{\circ}$. (b) Atom profiles for several tip-atom magnetization angles showing the gradual development of shape asymmetry, defined as the imbalance between the light and dark gray areas enclosed by the atom profile, Eq. (3). (c)-(d) Comparison of constant-current (c) and constant-height (d) modes $(0 \mathrm{~T}, 1.1 \mathrm{~K}$, the set point for scanning or feedback opening is $10 \mathrm{mV}$ and $2 \mathrm{nA}$ ). Note that the color scale is adapted in each image to highlight that the atoms have the same shape in spite of having enhanced magnetic contrast in constant-height mode. (e) Profiles extracted from (c) and (d) illustrating that the spin-dependent shape in closed feedback (i.e., constant-current mode, black line) is identical to constant-height LDOS slices (pale blue line). Atomic profiles are vertically offset.

data are typically acquired in closed feedback mode because of a much easier technical implementation. In this mode, the contrast in tunneling magnetoconductance is transferred by the feedback loop to topographic contrast to keep the total current constant. In this process, the feedback introduces a nonlinear relationship between the LDOS variations and the resulting topography. As a consequence, in the constant-current mode, Eq. (1) becomes an approximation of the experimental magnetic height contrast. The interpretation of height contrast as magnetoconductance is particularly well suited when looking at magnetic periodic patterns $[7,18,21,22]$ or bimodal contrast between states with opposite spin moment $[1,23]$. However, we are dealing with the shape of the atom's LDOS in a quantitative manner, and the use of Eq. (1) ought to be supported by a comparison with open feedback SP-STM images, in which a spin-resolved LDOS slice is accessed by keeping the $z$ component of $\mathbf{r}$ constant.

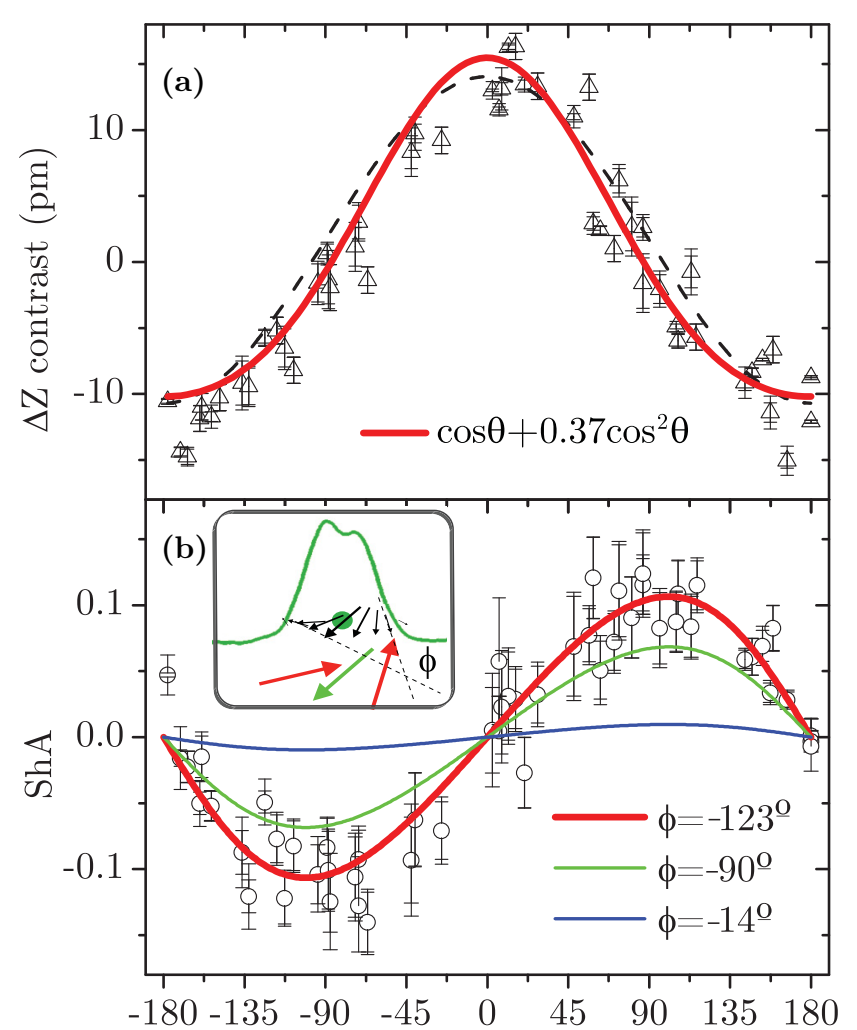

Angle between tip and adatom magnetization (deg)

FIG. 3. (a) Topographic spin-polarized height contrast and its corresponding $\cos \theta+\eta \cos ^{2} \theta$ fit with $\eta=0$ (thin dashed line) and $\eta=0.37$ (thick red line). (b) Experimental shape asymmetry of the atoms with a fit to Eq. (4), yielding $\phi=-123 \pm 3^{\circ}$ (red). Error bars are derived from the uncertainty in the atom's center position of $\pm 0.2 \AA$. The inset illustrates the spin density across the atom introduced by the linear dependence of $\theta(x)$ (see text), and the corresponding asymmetric height profile from which $\mathrm{ShA}$ is retrieved.

The comparison is shown in Figs. 2(c) (closed feedback) and 2(d) (open feedback), obtained under identical conditions. Following the results in Fig. 3, we arranged three atoms with maximum magnetic height contrast $\left(\theta_{\mathrm{Co}} \simeq 0^{\circ}, 180^{\circ}\right)$ and maximum ShA contrast $\left(\theta_{\mathrm{Co}} \simeq-90^{\circ}\right)$. The main difference between both modes is that in closed feedback the relative change of height between opposite spins is 0.7 times lower than the magnetoconductance ratio in open feedback. In other words, the feedback damps the spin-dependent contrast. This introduces an angle-dependent factor relating the contrast in $Z$ and $I$. Furthermore the $I$ atoms' profiles are narrower than the $Z$ ones by an angle-independent scaling factor in the distance, i.e., $Z(x) \propto I(0.72 x)$ [Fig. 2(e)]. However, it can be readily shown that the form of Eq. (3) cancels out these differences, and makes the value of ShA insensitive to the STM operation mode. Figure 2(e) illustrates that the atomic shape asymmetry is indeed preserved with great accuracy in closed feedback mode.

Thus, having shown that $Z$ can be replaced by $I$ as ShA concerns, we can insert Eq. (1) to calculate an explicit form of $\operatorname{ShA}\left(\theta_{\mathrm{Co}}\right)$. We will further assume that the atom's LDOS is constant in a small energy window around the Fermi level $(|e V|<10 \mathrm{meV})$ so that $\tilde{m}_{s}(\mathbf{r}, V)=m_{s}(x)$ and $\tilde{\rho}_{s}(\mathbf{r}, V)=$ 
$\rho_{s}(x)$. The central result of this work stems from the fact that the adatom's LDOS inherits the magnetic noncollinearity of the Mn SS as a consequence of hybridization of the atom's wave function with the substrate. The breaking of the translational symmetry of the spin space within the atom can be introduced, in its simplest form, as a continuous linear variation of the magnetic quantization axis above the adsorbed object [24]: $\theta\left(x_{[11 \overline{1} 0]}\right)-\theta_{\mathrm{Co}}=\phi\left(x-x_{\mathrm{Co}}\right) / w, x_{\mathrm{Co}}$ being the position of the atom center and $\phi / w$ represents the rotation pitch of the quantization axis within the atom's apparent size. The inset in Fig. 3(b) schematically shows the spin density vector above the Co atom. Note that in this notation, the noncollinearity is absorbed in the $\theta(\mathbf{r})$ dependence, and therefore $m_{s}(x)$ is an even function. Then,

$$
\begin{gathered}
\operatorname{ShA}\left(\theta_{\mathrm{Co}}, a\right)=\frac{A \sin \theta_{\mathrm{Co}}}{1+B \cos \theta_{\mathrm{Co}}}, \\
A \equiv-\frac{2 P_{T}}{\int_{-a}^{a} \rho_{s}} \int_{0}^{a} m_{s}(x) \sin \left(\frac{\phi}{w} x\right) d x, \\
B \equiv \frac{2 P_{T}}{\int_{-a}^{a} \rho_{s}} \int_{0}^{a} m_{s}(x) \cos \left(\frac{\phi}{w} x\right) d x,
\end{gathered}
$$

and using again Eq. (1), $A$ and $B$ can be conveniently expressed as a function of the conductance profiles, $I_{\uparrow} \equiv I\left(x, \phi, \theta_{\mathrm{Co}}=\right.$ $\left.0^{\circ}\right)$ and $I_{\downarrow} \equiv I\left(x, \phi, \theta_{\mathrm{Co}}=180^{\circ}\right)$. These functions are experimentally determined from the constant-height data in Figs. 2(d) and 2(e). Provided that $P_{T} \neq 0$ and $a \phi / w<90^{\circ}$, the factors in Eq. (4) take the form

$$
\begin{gathered}
A=-\frac{2}{\int_{-a}^{a} I_{\uparrow}+I_{\downarrow}} \int_{0}^{a}\left(I_{\uparrow}-I_{\downarrow}\right) \tan \left(\frac{\phi}{w} x\right) d x, \\
B=\frac{2}{\int_{-a}^{a} I_{\uparrow}+I_{\downarrow}} \int_{0}^{a}\left(I_{\uparrow}-I_{\downarrow}\right) d x .
\end{gathered}
$$

It is remarkable that these expressions can be experimentally evaluated without the knowledge of the tip's spin polarization $\left(P_{T}\right)$ or the collinear magnetic moment density $\left(m_{s}\right)$. The best fit of ShA data in Fig. 3(b) to Eq. (4) is obtained for $\phi=-123 \pm 10^{\circ}$. We have used $a=2.5 \AA$, although the sensitivity of the measured ShA to $a$ is negligible. The necessary condition leading to magnetic shape contrast is that $\phi \neq 0$, i.e., the magnetic ground state is noncollinear. On the other hand, a marked orbital contrast in the spin-up and spin-down subbands, as is the case for $\mathrm{Co} / \mathrm{Mn} / \mathrm{W}(110)$ [9], brings up subatomic features in $m_{s}(x)$ away from $x=0$, increasing the value of $A$ [see Eq. (5)]. We note that we have assumed a rotation angle linear with the distance from the atoms' center, and so the obtained value of $\phi$ represents an effective model-dependent parameter.

Although we now have a clear picture of the scenario for states at the Fermi level, there is still the question whether the concept of magnetic shape asymmetry will also apply to a broader bias range. As sample bias increases in absolute value, the $\tilde{\mathbf{m}}_{s}$, as well as orbital contrast, will cancel out because the features of the LDOS up to $|e V|$ are averaged. This is illustrated in Fig. 4(a), where we represent a collection of Co atoms positioned with equispaced $\theta_{\mathrm{Co}}$. Here, the topographic spin contrast at $-100 \mathrm{meV}$ remains in the apparent height but has almost dissapeared in the shape. Instead, one can make
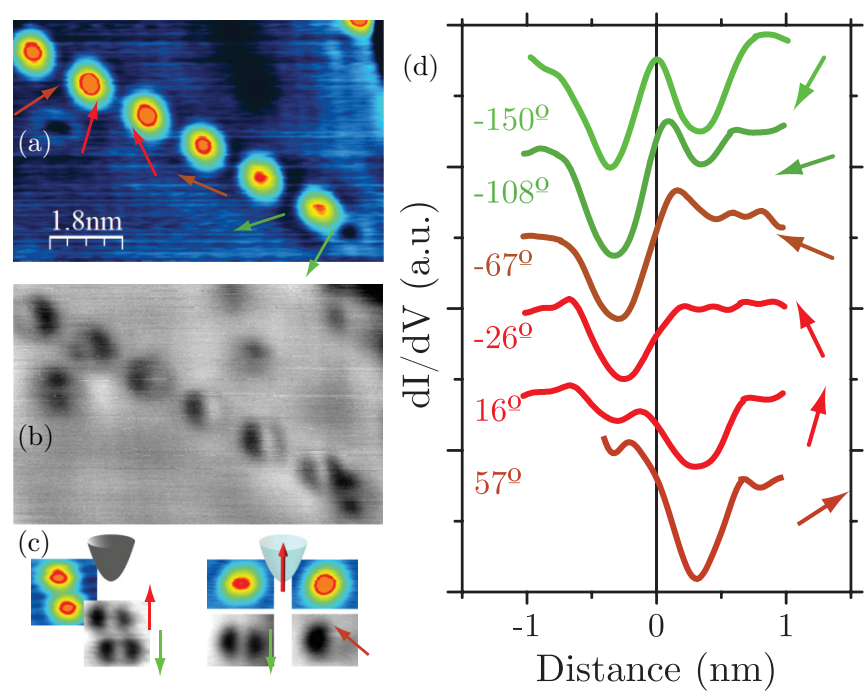

FIG. 4. (a) Spin-resolved constant-current map and (b) $d I / d V$ conductance map of Co atoms positioned every $3 w$ along the spin spiral, leading to $\Delta \theta_{\mathrm{Co}} \simeq-42^{\circ}(2 \mathrm{nA}$, sample bias $-100 \mathrm{mV}$, lock-in modulation $5 \mathrm{mV}$ and $+2.5 \mathrm{~T}$ ). (c) Constant-current and $d I / d V$ map over two atoms with opposite spin (that is, separated by $w / 2$ in the horizontal axis) scanned with a nonmagnetic W tip (left panel) and two atoms with almost opposite spin scanned with a Fe-coated W tip (right panel). This set of images shows that at $-100 \mathrm{meV}$ the vacuum LDOS of Co atoms exhibits a strong $d_{x z}$ orbital contribution and is dominated by the minority spin channel. Since $d I / d V$ mapping is energy selective, at $-100 \mathrm{meV}$, the distinct nonspherical orbital symmetry contrasts with the round-shaped atom topography responding to the total tunneling current. (d) Enhanced spin-shape asymmetry in atoms' profiles along [110] from $d I / d V$ conductance maps at $-100 \mathrm{mV}$.

use of the spectroscopy capabilities of STM by recording $d I / d V$ maps at a particular bias $V$ by means of the lock-in technique, which represents a signal proportional to the LDOS at energy $e V$. The $d I / d V$ map at $e V=-100 \mathrm{meV}$ recovers the orbital contrast [see Fig. 4(b)] and displays a strong spin-dependent asymmetry between the left and right sides of the atoms. Spin-averaging maps taken with a bare W tip of Co/Mn/W(110), shown in Fig. 4(c) (left), reveal an LDOS with a dominant double-lobe structure having the symmetry of the $d_{x z}$ orbital, independently of $\theta_{\mathrm{Co}}$. At the same time, spin-resolved images of Co atoms with almost opposite spin [Fig. 4(c), right] show that the spin-down LDOS resembles closely the total LDOS [Fig. 4(c) left], and that the spin-up LDOS has a different spatial distribution which damps the contrast between the two lobes [25]. This can be explained by a large spin polarization combined with a marked $d_{x z}$ character of the spin-down LDOS. Therefore, the Co spin moment is also primarily carried by the $d$-shell electrons, as in the case of the near Fermi level LDOS, but with a greater negative spin polarization at $-100 \mathrm{meV}$. As a consequence, and according to Eqs. (4) and (5), the asymmetry induced by the substrate noncollinearity should also be enhanced. This is consistent with the $d I / d V$ profiles shown in Fig. 4(d), where a large positive asymmetry appears for atoms with $\theta_{\mathrm{Co}}=57^{\circ}$ (close to $90^{\circ}$ ) and large negative asymmetry for atoms with $\theta_{\mathrm{Co}}=-108^{\circ}$ (close to $-90^{\circ}$ ). Not only the sign of 
the asymmetry is preserved, but also the monotonous variation as a function of $\theta_{\mathrm{Co}}$.

The physical concept reported here can be of general application in a large variety of magnetic substrates with noncollinear ground states: $2 \mathrm{AL} \mathrm{Fe} / \mathrm{Cu}(111)$ [26], $2 \mathrm{AL} \mathrm{Mn/W(110)} \mathrm{[22],} 1 \mathrm{AL} \mathrm{Mn/W(100)} \mathrm{[27],} 1 \mathrm{AL}$ Mn/Ag(111) [21], 2 AL Fe/W(110) [28], 1 AL Fe/Ir(111) [29], and NiMn(001) [30]. This work opens a way to study not only the coupling of adatoms to those substrates, but also the possible magnetic interactions among atoms in artificial structures built by atomic manipulation. We would like to highlight that the role of an external magnetic field here is restricted to validating the magnetic origin of the observed contrast. In addition, the applied magnetic field in combination with soft Fe-coated W-tips permits us to know the spin-sensitivity direction in real space, but in practice the whole analysis has been carried out in the reference quantization axis of the tip. The conclusions are valid for any tip magnetization direction, and therefore this sort of experiment can be conducted in the absence of an external magnetic field. We envisage the implementation of spin-shape asymmetry as a versatile tool for performing magnetic studies sensitive to two orthogonal spin directions at the scale of individual atoms.

To conclude, we have demonstrated that single atoms coupled to a noncollinear magnetic state exhibit a spin- dependent shape asymmetry. In the case of Co atoms on $\mathrm{Mn} / \mathrm{W}(110)$, the shape asymmetry is proportional to the spin projection orthogonal to the direction probed in conventional SP-STM experiments. In this way the $\theta /-\theta$ degeneracy of Eq. (1) is removed, allowing the full determination of the angle between tip and adatom magnetizations. The effect appears both in topography at low bias or in $d I / d V$ data at selected energies, and can be quantitatively determined under closed feedback conditions. Also, we have demonstrated an unprecedented level of spectroscopic information combining spin, orbital, and energy resolution. High spatial resolution of spin-split molecular orbitals has been obtained in some small metal-organic compounds exhibiting spin-dependent contrast [31,32] with magnetic tips. However, this study presents results of spin-resolved orbital imaging of single-atom wave functions.

We thank Kirsten von Bergmann, Paolo Ferriani, and Stephan Heinze for stimulating discussions. We acknowledge financial support provided by the DFG Sonderforschungbereich SFB 668, the Plan Nacional de I+D+i (Grants No. MAT2010-19236 and No. MAT2013-46593-C6-3-P), and the Marie Curie Programme (MEIF-CT-2006-039071). D.S. and M.M.L. acknowledge the use of Servicio General de Apoyo a la Investigación (University of Zaragoza).
[1] F. Meier, L. Zhou, J. Wiebe, and R. Wiesendanger, Science 320, 82 (2008).

[2] Y.-S. Fu, T. Zhang, S.-H. Ji, X. Chen, X.-C. Ma, J.-F. Jia, and Q.-K. Xue, Phys. Rev. Lett. 103, 257202 (2009).

[3] R. Vincent, S. Klyatskaya, M. Ruben, W. Wernsdorfer, and F. Balestro, Nature 488, 357 (2012).

[4] I. G. Rau, S. Baumann, S. Rusponi, F. Donati, S. Stepanow, L. Gragnaniello, J. Dreiser, C. Piamonteze, F. Nolting, S. Gangopadhyay et al., Science 344, 988 (2014).

[5] S. Yan, D.-J. Choi, J. A. J. Burgess, S. Rolf-Pissarczyk, and S. Loth, Nat. Nanotech. 10, 40 (2015).

[6] A. A. Khajetoorians, J. Wiebe, B. Chilian, and R. Wiesendanger, Science 332, 1062 (2011).

[7] S. Loth, S. Baumann, C. P. Lutz, D. M. Eigler, and A. J. Heinrich, Science 335, 196 (2012).

[8] R. Wiesendanger, H.-J. Güntherodt, G. Güntherodt, R. J. Gambino, and R. Ruf, Phys. Rev. Lett. 65, 247 (1990).

[9] D. Serrate, P. Ferriani, Y. Yoshida, S.-W. Hla, M. Menzel, K. von Bergmann, S. Heinze, A. Kubetzka, and R. Wiesendanger, Nat. Nanotech. 5, 350 (2010).

[10] M. Menzel, Y. Mokrousov, R. Wieser, J. E. Bickel, E. Vedmedenko, S. Blügel, S. Heinze, K. von Bergmann, A. Kubetzka, and R. Wiesendanger, Phys. Rev. Lett. 108, 197204 (2012).

[11] M. Jullière, Phys. Lett. A 54, 225 (1975).

[12] J. C. Slonczewski, Phys. Rev. B 39, 6995 (1989).

[13] S. Yuasa, T. Nagahama, A. Fukushima, Y. Suzuki, and K. Ando, Nat. Mater. 3, 868 (2004).

[14] S. Parkin, C. Kaiser, A. Panchula, P. Rice, B. Hughes, M. Samant, and S. H.Yung, Nat. Mater. 3, 862 (2004).
[15] D. Wortmann, S. Heinze, P. Kurz, G. Bihlmayer, and S. Blügel, Phys. Rev. Lett. 86, 4132 (2001).

[16] M. Bode, M. Heide, K. von Bergmann, P. Ferriani, S. Heinze, G. Bihlmayer, A. Kubetzka, O. Pietzsch, S. Blügel, and R. Wiesendanger, Nature 447, 190 (2007).

[17] I. Horcas, R. Fernández, J. M. Gómez-Rodríguez, J. Colchero, J. Gómez-Herrero, and A. M. Baró, Rev. Sci. Instrum. 78, 013705 (2007).

[18] K. von Bergmann, M. Menzel, D. Serrate, Y. Yoshida, S. Schröder, P. Ferriani, A. Kubetzka, R. Wiesendanger, and S. Heinze, Phys. Rev. B 86, 134422 (2012).

[19] N. M. Caffrey, S. Schröder, P. Ferriani, and S. Heinze, J. Phys. Condens. Matter 26, 394010 (2014).

[20] N. Neel, S. Schröder, N. Ruppelt, P. Ferriani, J. Kröger, R. Berndt, and S. Heinze, Phys. Rev. Lett. 110, 037202 (2013).

[21] C. L. Gao, W. Wulfhekel, and J. Kirschner, Phys. Rev. Lett. 101, 267205 (2008).

[22] Y. Yoshida, S. Schröder, P. Ferriani, D. Serrate, A. Kubetzka, K. von Bergmann, S. Heinze, and R. Wiesendanger, Phys. Rev. Lett. 108, 087205 (2012).

[23] S. Krause, L. Berbil-Bautista, G. Herzog, M. Bode, and R. Wiesendanger, Science 317, 1537 (2007).

[24] N. M. Caffrey, P. Ferriani, S. Marocchi, and S. Heinze, Phys. Rev. B 88, 155403 (2013).

[25] This atom has a tilted spin of $\theta=-50^{\circ}$, and as such the appearance of the pure spin-up channel cannot be retrieved from it with a spin polarized tip.

[26] S. H. Phark, J. A. Fischer, M. Corbetta, D. Sander, K. Nakamura, and J. Kirschner, Nat. Commun. 5, 5183 (2014). 
[27] P. Ferriani, K. von Bergmann, E. Y. Vedmedenko, S. Heinze, M. Bode, M. Heide, G. Bihlmayer, S. Blügel, and R. Wiesendanger, Phys. Rev. Lett. 101, 027201 (2008).

[28] S. Meckler, N. Mikuszeit, A. Preßler, E. Y. Vedmedenko, O. Pietzsch, and R. Wiesendanger, Phys. Rev. Lett. 103, 157201 (2009).

[29] S. Heinze, K. von Bergmann, M. Menzel, J. Brede, A. Kubetzka, R. Wiesendanger, G. Bihlmayer, and S. Blügel, Nat. Phys. 7, 713 (2011).
[30] C. L. Gao, A. Ernst, A. Winkelmann, J. Henk, W. Wulfhekel, P. Bruno, and J. Kirschner, Phys. Rev. Lett. 100, 237203 (2008).

[31] J. Brede, N. Atodiresei, S. Kuck, P. Lazić, V. Caciuc, Y. Morikawa, G. Hoffmann, S. Blügel, and R. Wiesendanger, Phys. Rev. Lett. 105, 047204 (2010).

[32] J. Schwöbel, Y. Fu, J. Brede, A. Dilullo, G. Hoffmann, S. Klyatskaya, M. Ruben, and R. Wiesendanger, Nat. Commun. 3, 953 (2012). 